\title{
A fluid-dynamical model for 'anti-surfactants'
}

\author{
Justin J. A. Conn, Brian R. Duffy, David Pritchard, ${ }^{*}$ and Stephen K. Wilson \\ Department of Mathematics and Statistics, University of Strathclyde, \\ 26 Richmond Street, Glasgow G1 $1 X H$, Scotland, UK \\ Peter J. Halling \\ Department of Pure and Applied Chemistry, University of Strathclyde, \\ 295 Cathedral Street, Glasgow G1 1XL, Scotland, UK \\ Khellil Sefiane \\ School of Engineering, University of Edinburgh, The King's Buildings, \\ Mayfield Road, Edinburgh EH9 3JL, Scotland, UK
}

(Dated: February 20, 2016)

\begin{abstract}
We construct a fluid-dynamical model for the flow of a solution with a free surface at which surface tension acts. This model can describe both classical surfactants, which decrease the surface tension of the solution relative to that of the pure solvent, and 'anti-surfactants' (such as many salts when added to water, and small amounts of water when added to alcohol) which increase it. We demonstrate the utility of the model by considering the linear stability of an infinitely deep layer of initially quiescent fluid. In particular, we predict the occurrence of a novel instability driven by surface-tension gradients, which occurs for anti-surfactant, but not for surfactant, solutions.
\end{abstract}

\section{INTRODUCTION}

The surface tension of a solution generally differs from that of the pure solvent. The molecules or ions of many solutes accumulate preferentially at free surfaces, where they lower the surface tension [1]; such substances are consequently known as surfactants. However, it is also well known that there are other solutes that have the opposite effect: as increasing amounts of these solutes are added to the solvent, the surface tension increases. Examples include many salts when added to water $[1,2]$, and small amounts of water when added to alcohol [3]. For fluid-dynamical purposes, such solutes may conveniently be described as 'anti-surfactants'.

Since this anti-surfactant behaviour may play a significant role in the flow of solutions with free surfaces, it is perhaps surprising that it has not yet been incorporated into fluid-dynamical models, especially as corresponding models for both soluble and insoluble surfactants are now well established and have been widely studied [4-8]. It should be noted that, in general, a model for an antisurfactant cannot be obtained from one for a surfactant simply by reversing the dependence of the surface tension on the surface concentration of solute, because, as we shall explain below, this is not in general consistent with the underlying physical mechanisms by which solutes affect surface tension.

In this contribution we construct a fluid-dynamical model which builds on existing models for surfactants but which, unlike them, can also describe anti-surfactants. By considering a simple linear stability problem we demonstrate that the new model is tractable and predicts

* Corresponding author: david.pritchard@strath.ac.uk a novel instability driven by surface-tension gradients, which occurs for anti-surfactant, but not for surfactant, solutions.

\section{MODEL CONSTRUCTION}

\section{A. Bulk-surface flux and surface tension}

We follow many well-established fluid-dynamical models of coupled flow and surfactant transport [4-8] by distinguishing between the surface region of the fluid, taken to have a notional thickness $\eta$ of the order of ångströms, and the remaining bulk region of the fluid. The bulk region may include a 'subsurface' region of high concentration gradients, which mediates solute exchange between the surface and the deeper regions of the fluid [9]; for simplicity we assume that such a region may be described by the same governing equations as the rest of the bulk. The bulk concentration of solute $c_{\mathrm{b}}$ is measured in $\mathrm{mol} \mathrm{m}^{-3}$, while the surface concentration of solute $c_{\mathrm{s}}$ is measured in $\mathrm{mol} \mathrm{m}^{-2}$. The concentration in each region obeys an appropriate transport equation, and the exchange of solute between the bulk and the surface is described by the bulk-surface flux $J$ measured in $\mathrm{mol} \mathrm{m}^{-2} \mathrm{~s}^{-1}$. When the flux is zero, $J=0$, the surface concentration is in equilibrium with the bulk concentration at the top of the bulk region; this is a good approximation when flow and transport processes are slow compared with the kinetics of bulk-surface exchange. We will refer to this state as 'bulk-surface equilibrium', noting that a system that is in bulk-surface equilibrium may still be evolving slowly through diffusion-controlled adsorption [9-11]. In the more general situation in which the flux is non-zero, $J \neq 0$, both kinetics and diffusion play a part in solute transfer between the bulk and the surface: this is some- 
times referred to as 'mixed-kinetic adsorption' [9].

It is usual in existing models of surfactants to treat the surface tension $\sigma$ as a decreasing function of the surface concentration $c_{\mathrm{s}}$. This approach was developed originally for insoluble surfactants [e.g. 4] and subsequently extended to soluble surfactants [e.g. 5-7]. However, as we shall see, in general $\sigma$ also depends on the bulk concentration $c_{\mathrm{b}}$ evaluated at the top of the bulk region, or, equivalently, on the surface excess $\Gamma$, defined as

$$
\Gamma=c_{\mathrm{s}}-\eta c_{\mathrm{b}},
$$

where again the concentration $c_{\mathrm{b}}$ is evaluated at the top of the bulk region. The sign of $\Gamma$ indicates whether the molecules of solute preferentially accumulate at the surface $(\Gamma>0)$, as they do for surfactants, or in the bulk $(\Gamma<0)$, as they do for anti-surfactants. Note that when the surface concentration is high relative to the bulk concentration (specifically, when $c_{\mathrm{s}} \gg \eta c_{\mathrm{b}}$ ) the surface excess $\Gamma$ is well approximated by $c_{\mathrm{s}}$, justifying the usual approach for surfactants. However, as we shall now describe, in general, and particularly for anti-surfactants, it is necessary to account for the dependence on $c_{\mathrm{b}}$, i.e. to distinguish between $\Gamma$ and $c_{\mathrm{s}}$.

Although the details of the electrochemical mechanisms that lead to the exclusion of particular species from the surface remain the topic of ongoing research $[12,13]$, the qualitative mechanism by which they affect the equilibrium surface tension is clear. Solvent molecules in the bulk interact with the dissolved solute; those near the surface have less interaction with the solute, as well as with other solvent molecules, and hence have higher energy than in the bulk, the excess being exhibited as surface energy. What determines the net effect of the added solute is then not the absolute values of $c_{\mathrm{S}}$ and $c_{\mathrm{b}}$, but the effective difference between these, i.e. the surface excess $\Gamma$ defined by (1).

More quantitatively, the surface excess plays a fundamental thermodynamic role described by the Gibbs isotherm $[1,9]$, which requires that in equilibrium the gradient of surface tension with respect to bulk concentration can be positive only if the surface excess is negative. For surfactants, the equilibrium surface excess is positive and so the equilibrium surface tension decreases with increasing bulk concentration; conversely, for antisurfactants the equilibrium surface excess must be negative to lead to the experimentally observed increase in the equilibrium surface tension with bulk concentration.

Starting from standard expressions for the bulksurface flux of solute, we may employ the Gibbs isotherm to obtain a relationship between surface tension and surface excess when bulk-surface equilibrium holds. We will then extend this relationship to situations in which bulk-surface equilibrium does not hold. The new fluiddynamical model that emerges is able to capture both surfactant and anti-surfactant behaviour, and thus allows us to explore the essential differences between flows driven by surfactants and flows driven by antisurfactants.
We now describe the simplest model that may be constructed within this framework. Incorporating other effects, such as a maximum surface concentration due to packing effects or a critical micelle concentration in the bulk [7], is straightforward in principle but in practice it introduces distracting complications and so is not discussed further here. Similarly, we do not attempt to represent the underlying molecular or ionic interactions that control the surface excess and its effects [13], but in the spirit of established surfactant models [4-8] we subsume these into a simple kinetic description.

We consider the bulk-surface flux

$$
J=k_{1} \eta c_{\mathrm{b}}-k_{2} c_{\mathrm{s}}
$$

for some adsorption and desorption rate constants $k_{1}$ and $k_{2}$. In bulk-surface equilibrium, $J=0$, equation (2) reduces to the Henry isotherm [9], i.e. $c_{\mathrm{s}}^{\mathrm{eq}}=K \eta c_{\mathrm{b}}^{\mathrm{eq}}$, where $K=k_{1} / k_{2}$ and the superscript 'eq' denotes an equilibrium value.

Using the definition (1), the equilibrium surface excess $\Gamma^{\text {eq }}$ is given by

$$
\Gamma^{\mathrm{eq}}=c_{\mathrm{s}}^{\mathrm{eq}}-\eta c_{\mathrm{b}}^{\mathrm{eq}}=(K-1) \eta c_{\mathrm{b}}^{\mathrm{eq}} .
$$

Under isothermal conditions, the Gibbs isotherm $[1,9]$ relates the equilibrium surface tension $\sigma^{\text {eq }}$ of a dilute solution to $\Gamma^{\mathrm{eq}}$ according to

$$
c_{\mathrm{b}}^{\mathrm{eq}} \frac{\mathrm{d} \sigma^{\mathrm{eq}}}{\mathrm{d} c_{\mathrm{b}}^{\mathrm{eq}}}=-R T \Gamma^{\mathrm{eq}},
$$

where $R$ denotes the gas constant and $T$ the (constant) temperature [14]. Substituting (3) into (4) and integrating with respect to $c_{\mathrm{b}}^{\mathrm{eq}}$ we obtain $\sigma^{\mathrm{eq}}$ in terms of $c_{\mathrm{b}}^{\mathrm{eq}}$,

$$
\sigma^{\mathrm{eq}}\left(c_{\mathrm{b}}^{\mathrm{eq}}\right)=\sigma_{0}+R T(1-K) \eta c_{\mathrm{b}}^{\mathrm{eq}},
$$

where $\sigma_{0}$ is the surface tension of pure solvent (i.e. $\sigma^{\text {eq }}=$ $\sigma_{0}$ when $\left.c_{\mathrm{b}}^{\text {eq }}=0\right)$. If $1-K<0$ then (5) corresponds to a surfactant for which $\sigma^{\text {eq }}$ decreases with $c_{\mathrm{b}}^{\text {eq }}$, whereas if $1-K>0$ then it corresponds to an anti-surfactant with the opposite behaviour [15]. The conditions $1-K \lessgtr 0$ correspond, respectively, to $k_{1} \gtrless k_{2}$, i.e. to conditions on the relative sizes of the adsorption and desorption rate constants.

In the absence of a thermodynamic theory for nonequilibrium surface tension, the equilibrium equation for $\sigma$ given by (5) can be extended to non-equilibrium situations in various ways. In general we may expect $\sigma$ to depend instantaneously on both $c_{\mathrm{b}}$ and $c_{\mathrm{s}}$, but we cannot expect there to be a non-equilibrum relation between them corresponding to the Henry isotherm. In principle, any function that reduces to (5) in equilibrium could be considered but, following the usual modelling principle that the model should be the simplest one capable of capturing the key physical mechanisms, we consider a general linear surface-tension law

$$
\sigma=\sigma_{0}+R T(1-K)\left(\frac{1-\theta}{K} c_{\mathrm{s}}+\eta \theta c_{\mathrm{b}}\right),
$$


where $\theta$ is an artificial parameter which is included in order to allow us to explore the sensitivity of our model to the relative importance of $c_{\mathrm{s}}$ and $c_{\mathrm{b}}$. As required, in bulk-surface equilibrium (i.e. when $J=0$ and hence $c_{\mathrm{s}}=$ $K \eta c_{\mathrm{b}}$ ), equation (6) reduces to $\sigma=\sigma_{0}+R T(1-K) \eta c_{\mathrm{b}}$ for any value of $\theta$. The particular choice $\theta=1$ makes $\sigma$ a function of $c_{\mathrm{b}}$ only, the particular choice $\theta=0$ makes $\sigma$ a function of $c_{\mathrm{s}}$ only, recovering the equation used in the standard surfactant models, while the particular choice $\theta=1 /(1-K)$ makes $\sigma$ a function of the surface excess $\Gamma$ only. By considering all three of these choices, we will demonstrate that our stability results are not qualitatively sensitive to the value of $\theta$, and thus that they are not an artefact of the details of the specific surfacetension law chosen. We will, however, demonstrate that the choice of $\theta$ may have experimentally observable consequences. Of course, within the confines of the linear stability analysis described in $\S$ III below, essentially any choice of the functional form of the surface-tension law will reduce to a linear expression and so, at least as far the linear stability results are concerned, the expression used in (6) is completely general.

\section{B. Hydrodynamics and solute transport}

Having obtained equation (6) for the surface tension $\sigma$, we incorporate it into a standard hydrodynamic model based on the Navier-Stokes equations along with advection-diffusion equations for solute transport.

The governing equations are

$$
\begin{gathered}
\nabla \cdot \mathbf{u}=0 \\
\rho\left(\frac{\partial \mathbf{u}}{\partial t}+(\mathbf{u} \cdot \nabla) \mathbf{u}\right)=-\nabla p+\mu \nabla^{2} \mathbf{u} \\
\frac{\partial c_{\mathrm{b}}}{\partial t}+(\mathbf{u} \cdot \nabla) c_{\mathrm{b}}=D_{\mathrm{b}} \nabla^{2} c_{\mathrm{b}} \\
\frac{\partial c_{\mathrm{s}}}{\partial t}+\left(\mathbf{u} \cdot \nabla_{2 \mathrm{D}}\right) c_{\mathrm{s}}+c_{\mathrm{s}}\left(\nabla_{\mathrm{s}} \cdot \mathbf{u}\right)=D_{\mathrm{s}} \nabla_{\mathrm{s}}^{2} c_{\mathrm{s}}+J
\end{gathered}
$$

where $\hat{\mathbf{n}}$ is the outward unit normal to the free surface, $\nabla_{\mathrm{s}}=\nabla-\hat{\mathbf{n}}(\hat{\mathbf{n}} \cdot \nabla)$ is the surface gradient operator, $\nabla_{2 \mathrm{D}}$ is an appropriate two-dimensional gradient operator [16], $\mathbf{u}$ and $p$ denote the velocity and pressure of the fluid, respectively, $t$ denotes time, $\rho$ and $\mu$ are the constant density and viscosity of the fluid, respectively, and $D_{\mathrm{b}}$ and $D_{\mathrm{s}}$ are the bulk and surface diffusivities, respectively. Note that (10) differs in the advective term from the transport equation derived by Stone [17] and used in many subsequent studies of surfactants; the corrected version of this equation employed here was derived by Wong et al. [18], and is used in more recent studies [e.g. 8]. For simplicity, body forces are neglected throughout, but they could readily be incorporated.

Equations (7)-(10) are to be solved subject to appropriate boundary conditions at the free surface $z=$ $h(x, y, t)$, namely

$$
\begin{gathered}
\frac{\partial h}{\partial t}+(\mathbf{u} \cdot \nabla)(h-z)=0, \\
\hat{\mathbf{n}} \cdot \mathbf{T} \cdot \hat{\mathbf{n}}=-\left(\nabla_{\mathrm{s}} \cdot \hat{\mathbf{n}}\right) \sigma, \\
\hat{\mathbf{n}} \cdot \mathbf{T} \cdot \hat{\mathbf{t}}=\hat{\mathbf{t}} \cdot \nabla_{\mathrm{s}} \sigma, \\
D_{\mathrm{b}} \hat{\mathbf{n}} \cdot \nabla c_{\mathrm{b}}=-J,
\end{gathered}
$$

where $\hat{\mathbf{t}}$ denotes any unit tangent vector lying in the tangent plane to the free surface, $\mathbf{T}$ is the total stress tensor, and all bulk quantities are evaluated on $z=h$.

Equations (7)-(14) with the bulk-surface flux $J$ given by (2) and the surface tension $\sigma$ given by (6) constitute our new fluid-dynamical model. This model can represent both surfactants and anti-surfactants, and can be 'tuned' through the choice of the parameter $\theta$ to represent different generalisations of the equation for the equilibrium surface tension (5) to non-equilibrium situations, including (as a special case) that used in the standard surfactant models.

\section{STABILITY OF AN INFINITELY DEEP LAYER}

We now consider a simple stability problem, which demonstrates that the new model (2), (6)-(14) is tractable and predicts a novel instability driven by surface-tension gradients, which occurs for antisurfactants but not for surfactants.

We consider the stability of an infinitely deep, initially quiescent layer of fluid with a flat free surface which, referred to the natural Cartesian coordinates $(x, y, z)$, occupies the region $z<0$, and is initially in bulk-surface equilibrium and at constant atmospheric pressure $p_{\mathrm{a}}$. The far-field conditions are taken to be

$$
\mathbf{u} \rightarrow \mathbf{0} \text { and } \quad \frac{\partial c_{\mathrm{b}}}{\partial z} \rightarrow 0 \quad \text { as } \quad z \rightarrow-\infty
$$

and hence the base state for the stability analysis is

$$
\mathbf{u}=\mathbf{0}, \quad p=p_{\mathrm{a}}, \quad c_{\mathrm{b}}=c_{\mathrm{b} 0}, \quad c_{\mathrm{s}}=\frac{k_{1} \eta}{k_{2}} c_{\mathrm{b} 0}, \quad h=0,
$$

where $c_{\mathrm{b} 0}$ is the uniform base-state value of $c_{\mathrm{b}}$. For simplicity, we will consider only two-dimensional perturbations, and neglect any variation or velocity in the $y$ direction.

We note that, by taking the depth of the fluid layer to be infinite, we exclude the possibility of finding solutions with a characteristic length scale proportional to the depth of the layer as in, for example, classical steady Marangoni convection [19, 20]. For surfactants, this is problematic, because all perturbations have this length scale; the infinite-depth problem is therefore degenerate. However, for anti-surfactants the perturbations with the greatest growth rate have a boundary-layer structure, and so do not degenerate in the limit of infinite depth. In 
the following analysis we will therefore generally confine the discussion to anti-surfactants, $0<K<1$; appendix A investigates the finite-depth problem, and explains the degeneracy in more detail for both $0<K<1$ and $K>1$.

\section{A. Non-dimensionalisation}

We non-dimensionalise the problem by choosing a natural scaling with a characteristic velocity scale $U$ and a characteristic length scale $L$ which reflects the following four assumptions. First, the flow will be driven principally by surface-tension gradients, which thus set the characteristic velocity scale so that the Marangoni number $M a=R T \eta c_{\mathrm{b} 0} /(\mu U)=1$, and hence $U$ is given by

$$
U=\frac{R T \eta c_{\mathrm{b} 0}}{\mu} .
$$

Second, the characteristic concentration scale is set by the bulk and surface concentrations in the base state. Third, there is an approximate balance between advective and diffusive transport, so that the bulk Peclet number $P_{\mathrm{b}}=U L / D_{\mathrm{b}}=1$, and hence $L$ is given by

$$
L=\frac{\mu D_{\mathrm{b}}}{R T \eta c_{\mathrm{b} 0}} .
$$

Finally, following recent work on surfactants [e.g. 7] we also assume that $D_{\mathrm{s}}=D_{\mathrm{b}}$, so that the surface Peclet number $P_{\mathrm{s}}=U L / D_{\mathrm{s}}=P_{\mathrm{b}}=1$.

The scaled quantities are therefore defined via

$$
\begin{gathered}
x=L x^{*}, \quad z=L z^{*}, \quad \mathbf{u}=(u, w)=U \mathbf{u}^{*}=U\left(u^{*}, w^{*}\right), \\
t=\frac{L}{U} t^{*}, \quad p-p_{\mathrm{a}}=\frac{\mu U}{L} p^{*}, \quad \mathbf{T}=\frac{\mu U}{L} \mathbf{T}^{*}, \\
c_{\mathrm{b}}=c_{\mathrm{b} 0} c_{\mathrm{b}}^{*}, \quad c_{\mathrm{s}}=\eta c_{\mathrm{b} 0} c_{\mathrm{s}}^{*}, \quad h=L h^{*} .
\end{gathered}
$$

With the scalings (19), the hydrodynamic equations (7) and (8) become

$$
\begin{aligned}
\nabla^{*} \cdot \mathbf{u}^{*} & =0 \\
\operatorname{Re}\left(\frac{\partial \mathbf{u}^{*}}{\partial t^{*}}+\left(\mathbf{u}^{*} \cdot \nabla^{*}\right) \mathbf{u}^{*}\right) & =-\nabla^{*} p^{*}+\nabla^{* 2} \mathbf{u}^{*}
\end{aligned}
$$

where the Reynolds number $R e=\rho U L / \mu$.

The bulk and surface concentration equations (9) and (10) become

$$
\begin{gathered}
\frac{\partial c_{\mathrm{b}}^{*}}{\partial t^{*}}+\left(\mathbf{u}^{*} \cdot \nabla^{*}\right) c_{\mathrm{b}}^{*}=\nabla^{* 2} c_{\mathrm{b}}^{*} \\
\frac{\partial c_{\mathrm{s}}^{*}}{\partial t^{*}}+\left(\mathbf{u}_{\mathrm{s}}^{*} \cdot \nabla_{2 \mathrm{D}}^{*}\right) c_{\mathrm{s}}^{*}+c_{\mathrm{s}}^{*}\left(\nabla_{\mathrm{s}}^{*} \cdot \mathbf{u}^{*}\right)=\nabla_{\mathrm{s}}^{* 2} c_{\mathrm{s}}^{*}+D a_{1}\left(K c_{\mathrm{b}}^{*}-c_{\mathrm{s}}^{*}\right),
\end{gathered}
$$

where the advective Damköhler number $D a_{1}=k_{2} L / U$, and, as before, $K=k_{1} / k_{2}$.
The surface boundary conditions (11)-(14) become

$$
\begin{gathered}
\frac{\partial h^{*}}{\partial t^{*}}+u^{*} \frac{\partial h^{*}}{\partial x^{*}}=w^{*}, \\
\hat{\mathbf{n}} \cdot \mathbf{T}^{*} \cdot \hat{\mathbf{n}}=-\left(\nabla_{\mathrm{s}}^{*} \cdot \hat{\mathbf{n}}\right)\left[\frac{1}{C a}+\frac{(1-\theta)(1-K)}{K} c_{\mathrm{s}}^{*}+\theta(1-K) c_{\mathrm{b}}^{*}\right], \\
\hat{\mathbf{n}} \cdot \mathbf{T}^{*} \cdot \hat{\mathbf{t}}=\hat{\mathbf{t}} \cdot \nabla_{\mathrm{s}}^{*}\left[\frac{(1-\theta)(1-K)}{K} c_{\mathrm{s}}^{*}+\theta(1-K) c_{\mathrm{b}}^{*}\right] \\
\hat{\mathbf{n}} \cdot \nabla^{*} c_{\mathrm{b}}^{*}=-D a_{2}\left(K c_{\mathrm{b}}^{*}-c_{\mathrm{s}}^{*}\right),
\end{gathered}
$$

where the capillary number $C a=\mu U / \sigma_{0}$ and the diffusive Damköhler number $D a_{2}=\eta L k_{2} / D_{\mathrm{b}}$, and where all bulk quantities are evaluated on $z^{*}=h^{*}$.

The far-field conditions (15) become

$$
\mathbf{u}^{*} \rightarrow \mathbf{0} \quad \text { and } \quad \frac{\partial c_{\mathrm{b}}^{*}}{\partial z^{*}} \rightarrow 0 \quad \text { as } \quad z^{*} \rightarrow-\infty .
$$

Finally, the base state (16) becomes

$$
\mathbf{u}^{*}=\mathbf{0}, \quad p^{*}=0, \quad c_{\mathrm{b}}^{*}=1, \quad c_{\mathrm{s}}^{*}=K, \quad h^{*}=0 .
$$

Substituting for $U$ and $L$ from (17) and (18), respectively, we are left with the dimensionless parameters

$$
\begin{array}{r}
R e=\frac{\rho D_{\mathrm{b}}}{\mu}, \quad K=\frac{k_{1}}{k_{2}}, \quad C a=\frac{R T \eta c_{\mathrm{b} 0}}{\sigma_{0}}, \\
D a_{1}=\frac{k_{2} \mu^{2} D_{\mathrm{b}}}{\left(R T \eta c_{\mathrm{b} 0}\right)^{2}}, \quad D a_{2}=\frac{\mu k_{2}}{R T c_{\mathrm{b} 0}},
\end{array}
$$

together with the parameter $\theta$.

For sodium chloride in water at room temperature [21], we have the approximate parameter values

$$
\begin{array}{r}
\rho \approx 10^{3} \mathrm{~kg} \mathrm{~m}^{-3}, \quad \mu \approx 10^{-3} \mathrm{~kg} \mathrm{~m}^{-1} \mathrm{~s}^{-1}, \\
D_{\mathrm{b}} \approx 2 \times 10^{-9} \mathrm{~m}^{2} \mathrm{~s}^{-1}, \quad \sigma_{0} \approx 7 \times 10^{-2} \mathrm{~kg} \mathrm{~s}^{-2},
\end{array}
$$

while for water in a short-chain alcohol at room temperature $[3,22,23]$, we have

$$
\begin{array}{r}
\rho \approx 8 \times 10^{2} \mathrm{~kg} \mathrm{~m}^{-3}, \quad \mu \approx 5 \times 10^{-4} \mathrm{~kg} \mathrm{~m}^{-1} \mathrm{~s}^{-1}, \\
D_{\mathrm{b}} \approx 10^{-9} \mathrm{~m}^{2} \mathrm{~s}^{-1}, \quad \sigma_{0} \approx 2 \times 10^{-2} \mathrm{~kg} \mathrm{~s}^{-2} .
\end{array}
$$

In both cases, $R \approx 8 \mathrm{~kg} \mathrm{~m}^{2} \mathrm{~s}^{-2} \mathrm{~mol}^{-1} \mathrm{~K}^{-1}$ and $T \approx$ $300 \mathrm{~K}$. In addition, we take the surface thickness $\eta \approx$ $10^{-9} \mathrm{~m}[9]$. The desorption rate constant $k_{2}$ is the parameter that can be stated with least certainty, because of the well-established difficulties in measuring kinetic parameters associated with rapid adsorption and desorption [24]. A rough upper bound is provided by the rate at which molecules or ions diffuse across a distance $\eta$ in the absence of potential barriers; this leads to $k_{2} \approx D_{\mathrm{s}} / \eta^{2}$ and thus to an upper bound of $k_{2} \approx 10^{10} \mathrm{~s}^{-1}$. Because of this uncertainty we will consider a wide range of values of $k_{2}$, and we will demonstrate that its magnitude does not generally have a critical effect on the stability of the 
layer. Finally, in order to see a substantial effect of the solute on surface tension, for sodium chloride in water we consider the regime in which the base concentration is a substantial fraction of the saturation concentration; we thus take $c_{\mathrm{b} 0} \approx 5 \times 10^{3} \mathrm{~mol} \mathrm{~m}^{-3}$, corresponding to a mass concentration of roughly $25 \%$ [21]. For water in alcohol, a similar value of $c_{\mathrm{b} 0}$ ensures that the surface-tensionconcentration relation remains roughly linear [3]. Thus, for both situations we have, to the nearest decimal order of magnitude,

$$
R e \approx 10^{-3}, \quad C a \approx 0.1, \quad D a_{1} \lesssim 0.1, \quad D a_{2} \lesssim 1
$$

To simplify the analysis, we will henceforth take the limit $R e=0$. The parameters $K$ and $\theta$ are expected to be of order unity.

\section{B. Linear stability analysis}

We define a perturbation parameter $\epsilon \ll 1$ and seek perturbations to the base state $(29)$ in the form

$$
\begin{aligned}
u^{*}=\epsilon u_{1}, & w^{*}=\epsilon w_{1}, \quad p^{*}=\epsilon p_{1}, \\
c_{\mathrm{b}}^{*}=1+\epsilon c_{\mathrm{b} 1}, & c_{\mathrm{s}}^{*}=K+\epsilon c_{\mathrm{s} 1}, \quad h^{*}=\epsilon h_{1} .
\end{aligned}
$$

In the usual manner, we now seek solutions of the form

$$
\begin{array}{cc}
u_{1}=\mathrm{e}^{s t^{*}} \mathrm{e}^{\mathrm{i} k x^{*}} U\left(z^{*}\right), & w_{1}=\mathrm{e}^{s t^{*}} \mathrm{e}^{\mathrm{i} k x^{*}} W\left(z^{*}\right), \\
p_{1}=\mathrm{e}^{s t^{*}} \mathrm{e}^{\mathrm{i} k x^{*}} P\left(z^{*}\right), & c_{\mathrm{b} 1}=\mathrm{e}^{s t^{*}} \mathrm{e}^{\mathrm{i} k x^{*}} C\left(z^{*}\right), \\
c_{\mathrm{s} 1}=\mathrm{e}^{s t^{*}} \mathrm{e}^{\mathrm{i} k x^{*}} C_{\mathrm{s}}, & h_{1}=\mathrm{e}^{s t^{*}} \mathrm{e}^{\mathrm{i} k x^{*}} H,
\end{array}
$$

where $k>0$ is the wavenumber of the perturbations and where the growth rate $s$ is to be determined.

The governing equations (20)-(22) become

$$
\begin{gathered}
\mathrm{i} k U+W^{\prime}=0, \\
\mathrm{i} k P+k^{2} U-U^{\prime \prime}=0, \\
P^{\prime}+k^{2} W-W^{\prime \prime}=0, \\
s C+k^{2} C-C^{\prime \prime}=0,
\end{gathered}
$$

while the surface concentration equation (23) reduces to the boundary condition

$$
s C_{\mathrm{s}}+K \mathrm{i} k U(0)+k^{2} C_{\mathrm{s}}-D a_{1}\left(K C(0)-C_{\mathrm{s}}\right)=0 .
$$

The surface boundary conditions (24)-(27) become

$$
\begin{gathered}
s H-W(0)=0, \\
2 W^{\prime}(0)-P(0)+k^{2} \sigma_{\mathrm{b}} H=0, \\
U^{\prime}(0)+\mathrm{i} k W(0)-\mathrm{i} k \theta(1-K) C(0) \\
-\mathrm{i} k \frac{(1-\theta)(1-K)}{K} C_{\mathrm{s}}=0, \\
C^{\prime}(0)+D a_{2}\left(K C(0)-C_{\mathrm{s}}\right)=0,
\end{gathered}
$$

where for convenience we have defined the dimensionless base surface tension as

$$
\sigma_{\mathrm{b}}=\frac{1}{C a}+1-K \text {. }
$$

Note that for $0<K<1$ (anti-surfactants), $\sigma_{\mathrm{b}}>0$. However, for $K>1$ (surfactants) the linear dependence of $\sigma$ on $c_{\mathrm{b}}$ must break down at higher concentrations, and so the validity of our model is restricted to initial concentrations such that $0<K<1+1 / C a$.

The far-field conditions (28) become

$$
U \rightarrow 0, \quad W \rightarrow 0, \quad C^{\prime} \rightarrow 0 \quad \text { as } \quad z^{*} \rightarrow-\infty .
$$

We can eliminate $P$ and $U$ from the hydrodynamic equations (36)-(38) to obtain

$$
P=\frac{1}{\mathrm{i} k}\left(U^{\prime \prime}-k^{2} U\right), \quad U=-\frac{1}{\mathrm{i} k} W^{\prime},
$$

and thus $W$ satisfies

$$
W^{(4)}-2 k^{2} W^{\prime \prime}+k^{4} W=0 .
$$

The most general solution of (48) consistent with the far-field conditions (46) is

$$
W\left(z^{*}\right)=\left(A_{1}+A_{2} z^{*}\right) \mathrm{e}^{k z^{*}},
$$

where $A_{1}$ and $A_{2}$ are arbitrary constants. Similarly, the most general solution of (39) consistent with the far-field conditions (46) is

$$
C\left(z^{*}\right)=A_{3} \mathrm{e}^{\xi z^{*}}
$$

where $A_{3}$ is an arbitrary constant and where $\xi=\sqrt{k^{2}+s}$ (for the usual definition of $\sqrt{\cdot}$ with a branch cut on the negative real axis). To satisfy the far-field condition, we require that $\Re(\xi)>0$; this is automatically the case for unstable modes with $\Re(s)>0$, and indeed it remains the case as long as $k^{2}+s \notin \mathbb{R}_{-}$. If $k^{2}+s \in \mathbb{R}_{-}$then there are no non-trivial solutions to (39) that decay in the far field. This restriction reflects the degeneracy discussed in appendix A.

The surface boundary conditions (41)-(44) become

$$
\begin{gathered}
s H-W(0)=0, \\
W^{\prime \prime \prime}(0)-3 k^{2} W^{\prime}(0)-k^{4} \sigma_{\mathrm{b}} H=0, \\
-W^{\prime \prime}(0)-k^{2} W(0)+k^{2} \theta(1-K) C(0) \\
+k^{2} \frac{(1-\theta)(1-K)}{K} C_{\mathrm{s}}=0, \\
C^{\prime}(0)+D a_{2}\left(K C(0)-C_{\mathrm{s}}\right)=0,
\end{gathered}
$$

while the surface concentration equation (40) becomes

$$
\xi^{2} C_{\mathrm{s}}-K W^{\prime}(0)-D a_{1}\left(K C(0)-C_{\mathrm{s}}\right)=0 .
$$

Substituting the solutions (49) and (50) into (51)-(55) produces a $5 \times 5$ system for the coefficients $A_{1}, A_{2}, A_{3}$, $C_{\mathrm{s}}$ and $H$. Requiring non-trivial solutions then yields the solvability conditions

$$
k^{4} \sigma_{\mathrm{b}}+2 k^{3} s=0
$$


or

$$
\begin{array}{r}
2 \xi^{3}+2 D a_{2} K \xi^{2}+\left[(K-1)(1-\theta) k+2 D a_{1}\right] \xi \\
-D a_{2} K(1-K) k=0 .
\end{array}
$$

These two conditions correspond to two distinct eigensolutions with different physical interpretations.

The condition (56) gives a growth rate $s=-\frac{1}{2} \sigma_{\mathrm{b}} k$, and corresponds to eigensolutions of the form

$$
W\left(z^{*}\right)=\frac{1}{2} \sigma_{\mathrm{b}} k\left(k z^{*}-1\right) H \mathrm{e}^{k z^{*}}, \quad C\left(z^{*}\right)=0, \quad C_{\mathrm{s}}=0,
$$

so this mode represents classical levelling under constant surface tension [25], with no variations in the concentration either in the bulk or on the free surface.

In contrast, the eigensolutions corresponding to the condition (57) have the form

$$
\begin{array}{r}
W\left(z^{*}\right)=k(1-K) \frac{D a_{2} K+(1-\theta) \xi}{2 K\left(\xi+D a_{2} K\right)} C_{\mathrm{s}} z^{*} \mathrm{e}^{k z^{*}}, \\
H=0, \quad C\left(z^{*}\right)=\frac{D a_{2} C_{\mathrm{s}}}{\xi+D a_{2} K} \mathrm{e}^{\xi z^{*}},
\end{array}
$$

where $C_{\mathrm{s}} \neq 0$. This mode represents the evolution of the system with an undeformed free surface in which the flow is driven entirely by surface-tension gradients [cf. 19].

\section{The eigenmodes with an undeformed free surface}

We now consider in more detail the modes corresponding to (57) and (59), recalling that we require $\Re(\xi)>0$ to satisfy the far-field condition, and that instability (i.e. $\Re(s)>0$ ) corresponds to $\Re\left(\xi^{2}\right)>k^{2}$.

It is straightforward to obtain numerical solutions to (57) and thus to plot the perturbation growth rates $s(k)$. Figure 1 illustrates the perturbation growth rates for various parameter values, including three values of the parameter $\theta$. In all cases in which instability occurs, it does so at rather small dimensionless wavenumbers, while typical maximum growth rates are of the order of $s=10^{-4}$ to $10^{-3}$; the corresponding dimensional timescales $L /(s U)$ for the instability to develop are therefore of the order of $10^{-8}$ to $10^{-7} \mathrm{~s}$. Changing the value of $\theta$ does not qualitatively affect the growth rates, but changing the ratio of the Damköhler numbers can alter the stability; we will investigate this further below.

Guided by the numerical evidence that $s \in \mathbb{R}$, we may postulate that the principle of the exchange of stabilities holds. This allows us to obtain marginal stability curves for various parameters simply by setting $s=0$, and thus $\xi=k$, in (57), and solving for the appropriate parameter. (We omit the details here for brevity.) Figure 2 shows typical results, for reference parameter values that correspond to the solid line in figure $1 \mathrm{~b}$.

A key feature of figures $2 \mathrm{a}-\mathrm{d}$ is that in each case the unstable region is largest when $k=0$. In other words, the transition to instability first occurs for long-wave perturbations, although within the unstable region the maximum growth rate generally occurs for a non-zero wavenumber (figure 1). We will use this result below to obtain an explicit stability criterion. Small values of $D a_{1}$ favour instability (figure 2 a), as do large values of $D a_{2}$ (figure $2 \mathrm{~b}$ ); in each case there is a critical value of $k$ beyond which no instability is possible. The situation for $K$ (figure $2 \mathrm{c}$ ) is more interesting: for a given wavenumber $k$, only a finite band of values of $K$ permit instability. This is reasonable in physical terms: as $K \rightarrow 1$ the antisurfactant properties of the solute are lost, whereas when $K=0$ the solute is completely excluded from the free surface, and so no surface advection is possible. We will see below that surface advection is an essential part of the instability mechanism. Finally, figure $2 \mathrm{~d}$ indicates that larger values of the parameter $\theta$ tend to suppress instability for non-zero $k$, but as $k \rightarrow 0$ no value of $\theta$ is sufficient to suppress the instability completely.

Equipped with these results we can now interpret figure 3, which shows the structure of a typical unstable perturbation. The bulk and surface concentration perturbations are in phase, with the bulk concentration perturbation confined to a boundary layer of thickness $\mathcal{O}(1 / k)$. Since $k$ is small, the lengthscale is considerably larger than $L$, and so diffusion is weak compared with advection. The flow along the free surface is divergent in the centre of the plot, where $c_{\mathrm{s}}$ and $c_{\mathrm{b}}$ have maxima, and convergent at the edges of the plot, where $c_{\mathrm{s}}$ and $c_{\mathrm{b}}$ have minima.

We can understand the structure of the perturbation as follows. Near the centre of the plot, where the perturbations to the surface and the bulk concentrations are negative, the surface tension is lowered since $0<K<1$ (anti-surfactant behaviour); similarly, the surface tension is higher at the edges of the plot. The resulting flow along the free surface is from the centre of the plot towards the edges, where the maxima of $c_{\mathrm{s}}$ and $c_{\mathrm{b}}$ occur. Surface concentration is advected outwards by this surface flow, reinforcing the negative perturbation to $c_{\mathrm{s}}$; the coupling between $c_{\mathrm{s}}$ and $c_{\mathrm{b}}$ means that the perturbation to $c_{\mathrm{s}}$ induces a corresponding perturbation to $c_{\mathrm{b}}$. Thus the perturbation reinforces itself and grows.

Opposing this positive feedback are the effects of diffusion and viscosity, which tend to eliminate perturbations, and (more subtly) the loss of solute from the surface. The instability mechanism relies on a substantial quantity of solute being present in the surface layer, because it is surface advection that causes solute to accumulate in regions of high surface tension; there is no mechanism by which advection in the bulk can do so. All other things being equal, the flux between bulk and surface will tend to reduce this accumulation over a dimensionless timescale $1 / D a_{1}$, and so higher values of $D a_{1}$ will tend to inhibit the instability. On the other hand, higher values of $D a_{2}$ mean that the bulk concentration will respond more rapidly to changes in the flux, and so higher values of $D a_{2}$ will tend to assist the instability. This competi- 

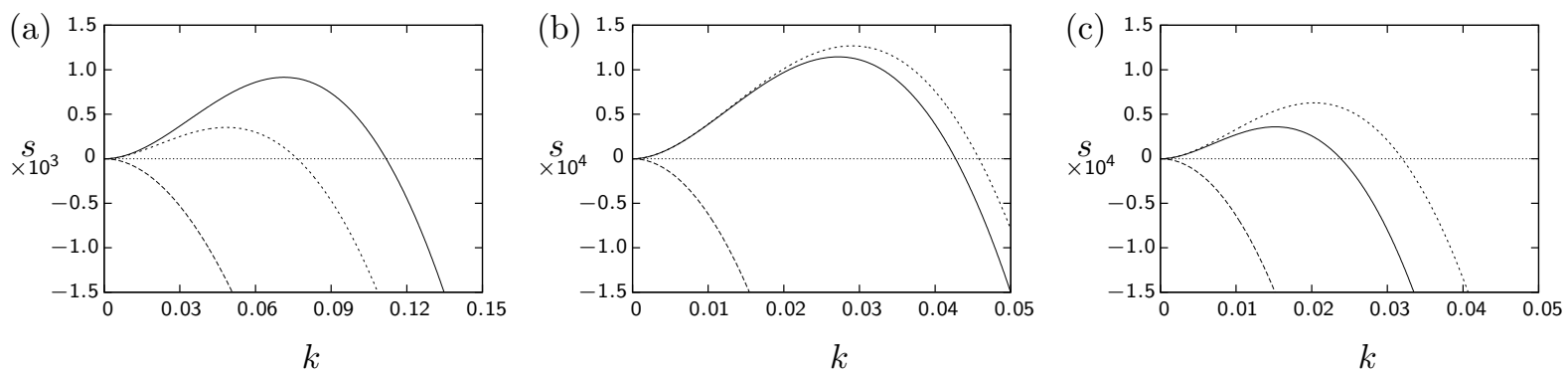

FIG. 1. Perturbation growth rates $s(k)$ for $K=0.5$ together with $D a_{1}=0.05, D a_{2}=0.5$ (solid), $D a_{1}=0.1, D a_{2}=0.5$ (heavy dashed), $D a_{1}=0.1, D a_{2}=1$ (light dashed), for (a) $\theta=0$, (b) $\theta=1$, (c) $\theta=2$.

(a)

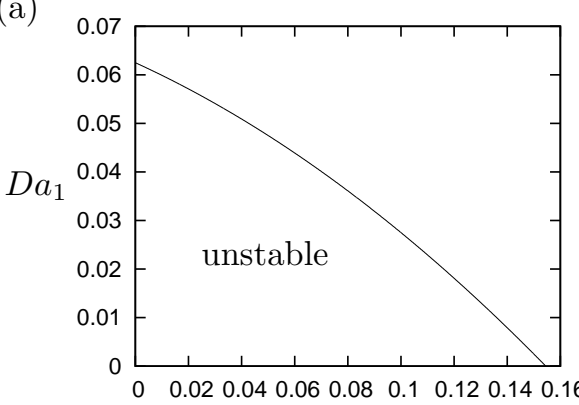

$k$

(c)

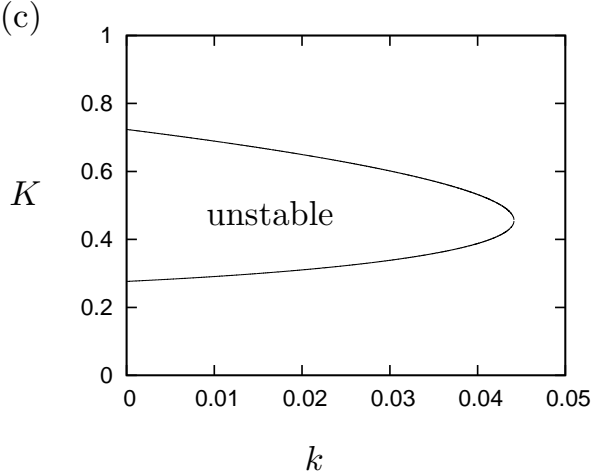

(b)

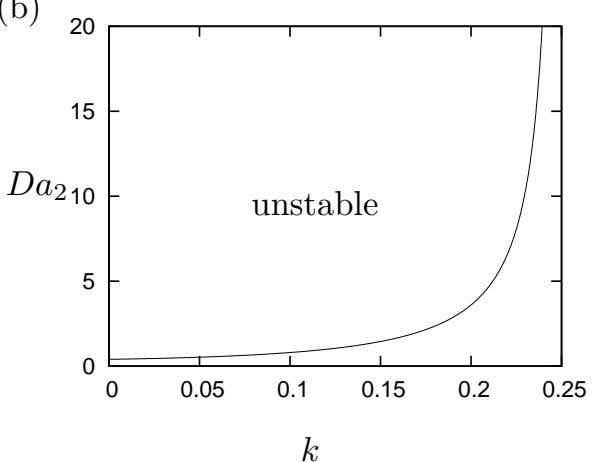

(d)

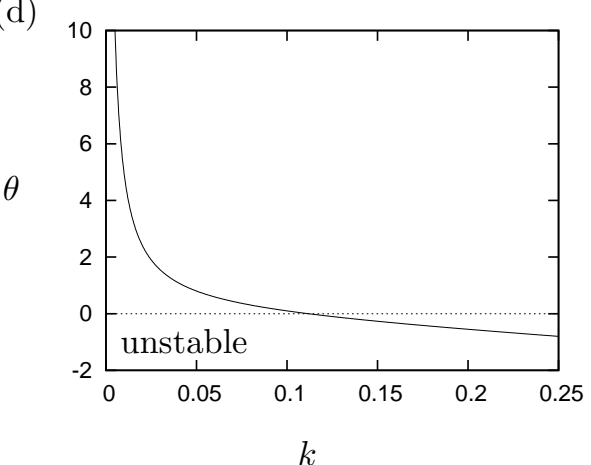

FIG. 2. Typical marginal curves in parameter space, for reference values $D a_{1}=0.05, D a_{2}=0.5, K=0.5$ and $\theta=1$. In each plot, one parameter is varied while the others are held constant at their reference values.

tion between the effects of increasing the two Damköhler numbers can be seen in figures $2 \mathrm{a}$ and $\mathrm{b}$, and explicitly in the long-wave stability criterion (65) derived below.

\section{The long-wave limit}

Motivated by figure 2, and further supported by the small range of $k$ for which instability occurs in figure 1 , we now investigate the long-wave limit $k \rightarrow 0^{+}$, in which the task of analysing the condition (57) becomes somewhat easier.

When $k=0$, the condition (57) reduces to the conditions $\xi=0$ or $\xi^{2}+D a_{2} K \xi+D a_{1}=0$; the latter has no solutions for which $\Re(\xi)>0$. Proceeding, we seek an asymptotic expansion of the form $\xi \propto k^{\alpha}$ for some $\alpha>0$, and it is straightforward to show by balancing terms that $\alpha=1$. This motivates the expansion

$$
\xi=\xi_{1} k+\xi_{2} k^{2}+\mathcal{O}\left(k^{3}\right),
$$

where $\Re\left(\xi_{1}\right)>0$ so that the condition $\Re(\xi)>0$ holds in this limit.

Substituting the expansion (60) into the condition (57) leads to

$$
\xi_{1}=\frac{D a_{2} K(1-K)}{2 D a_{1}}
$$




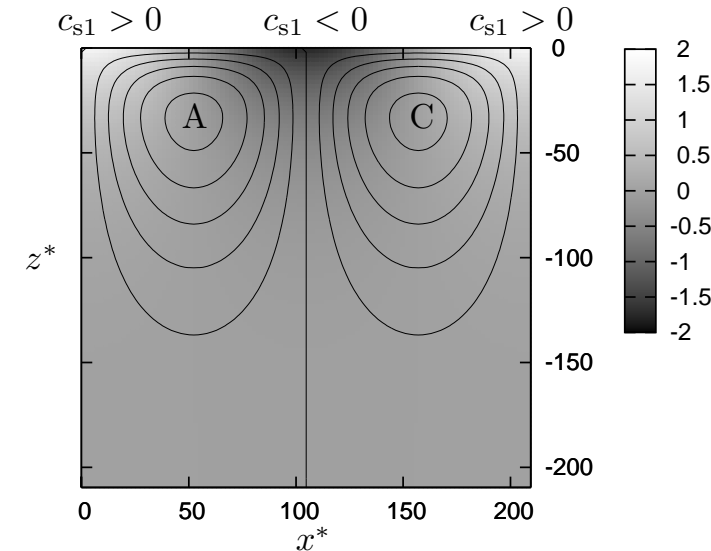

FIG. 3. Structure of a typical unstable perturbation for the parameter values used to plot figure $1 \mathrm{~b}$ with $k=0.03$, giving $s \approx 0.00011$. Greyscale shading represents the concentration perturbation $c_{\mathrm{b} 1}$, while the solid lines represent streamlines. The direction of flow is denoted by $\mathrm{C}$ (clockwise) or A (anticlockwise).

and

$$
\xi_{2}=-\frac{D a_{2} K(1-K)^{2}\left(D a_{2}^{2} K^{2}+D a_{1}(\theta-1)\right)}{4 D a_{1}^{3}} .
$$

When $0<K<1$, the coefficient $\xi_{1}$ is real and positive, and so the expansion remains consistent with the condition $\Re(\xi)>0$. However, when $K>1$ the expansion is no longer consistent with this condition. We therefore consider only the case $0<K<1$, corresponding to antisurfactants. (Appendix A discusses the case $K>1$ in more detail.)

Using (60), the expansion for $s=\xi^{2}-k^{2}$ becomes

$$
\begin{aligned}
s= & \left(\frac{D a_{2}^{2} K^{2}(1-K)^{2}}{4 D a_{1}^{2}}-1\right) k^{2} \\
& -\frac{D a_{2}^{2} K^{2}(1-K)^{3}\left(D a_{2}^{2} K^{2}+D a_{1}(\theta-1)\right)}{4 D a_{1}^{4}} k^{3}+\mathcal{O}\left(k^{4}\right) .
\end{aligned}
$$

The instability criterion for long waves $\left(k \rightarrow 0^{+}\right)$is thus

$$
\frac{D a_{2}^{2} K^{2}(1-K)^{2}}{4 D a_{1}^{2}}>1,
$$

which, recalling that $0<K<1$, reduces to

$$
\frac{D a_{2}}{D a_{1}}>\frac{2}{K(1-K)} .
$$

When $\xi_{2}>0$, we can also obtain an estimate for the typical unstable wavenumber,

$$
k_{\mathrm{typ}} \approx \frac{1-\xi_{1}^{2}}{\xi_{1} \xi_{2}}=\frac{2\left(D a_{2}^{2} K^{2}(1-K)^{2}-4 D a_{1}^{2}\right) D a_{1}^{2}}{D a_{2}^{2} K^{2}(1-K)^{3}\left(D a_{2}^{2} K^{2}+D a_{1}(\theta-1)\right)} .
$$

Numerically, for values of the parameters similar to those given in (33), $k_{\mathrm{typ}}$ is of the order of 0.04 , corresponding to dimensional wavelengths of the order of $2 \pi L / k \approx$ $3 \times 10^{-8} \mathrm{~m}$. This is small, but remains significantly larger than the surface layer thickness $\eta=10^{-9} \mathrm{~m}$, so the distinction between bulk and surface regions remains consistent.

We may rewrite the instability criterion (65) in terms of dimensional quantities as

$$
\frac{\eta \Delta \sigma_{\mathrm{b}}}{\mu D_{\mathrm{b}}}>\frac{2}{K},
$$

where we have written the difference between the surface tension in the base state and the surface tension of pure solvent as

$$
\Delta \sigma_{\mathrm{b}}=R T \eta c_{\mathrm{b} 0}(1-K) .
$$

It is useful to rearrange this further, noting that in equilibrium experiments bulk quantities rather than surface quantities are measured, and to write (67) as

$$
\frac{\Delta \sigma_{\mathrm{b}}}{R T \mu D_{\mathrm{b}}} \frac{\mathrm{d} \sigma}{\mathrm{d} c_{\mathrm{b}}}>\frac{2(1-K)}{K},
$$

where in our linear model $\mathrm{d} \sigma / \mathrm{d} c_{\mathrm{b}}=\Delta \sigma_{\mathrm{b}} / c_{\mathrm{b} 0}$. The lefthand side of (69) now consists solely of experimentally measurable quantities, while the right-hand side depends only on $K$, which in practice must be determined as a fitting parameter along with $\eta$. Since the right-hand side is a monotonically decreasing function of $K$, we conclude that the instability becomes easier to trigger the closer the value of $K$ becomes to $K=1$. A final but important point is that, since they enter (69) only through their ratio $K$, it is the relative rather than the absolute values of the adsorption and desorption rate constants $k_{1}$ and $k_{2}$ that affect the stability.

\section{The limit of small Damköhler numbers}

The Damköhler numbers used to plot figures 1-3 are not far below unity, and correspond to the upper end of the range of plausible values for the desorption rate constant $k_{2}$. Since, as previously discussed in $\S$ III A above, this rate constant could be several orders of magnitude smaller than its upper value, it is of interest to consider the predictions of the stability analysis for $0<K<1$ as the Damköhler numbers become small.

Figures $4 \mathrm{a}-\mathrm{c}$ illustrate typical perturbation growth rates for cases in which $\theta<1, \theta=1$ and $\theta>1$, respectively. (In figures $4 \mathrm{~b}$ and $\mathrm{c}$, the growth rates and 
wavenumbers have been scaled in a manner that will be discussed below.) The general behaviour of the perturbation growth rates is similar, but there is one difference, illustrated in figure 4 a: for $\theta<1$, as the Damköhler numbers become smaller the growth rate $s$ can become positive for intermediate wavenumbers even in cases where $s$ remains negative for smaller wavenumbers. We will discuss this further below.

To obtain analytical results, we consider the asymptotic limit in which both Damköhler numbers become small, while their ratio remains of order unity. Accordingly, we write $D a_{1}=\delta \hat{D}_{1}$ and $D a_{2}=\delta \hat{D}_{2}$ and consider the limit $\delta \rightarrow 0$ with $\hat{D}_{1}$ and $\hat{D}_{2}$ of order unity. Equation (57) becomes

$$
\begin{array}{r}
2 \xi^{3}+2 \delta \hat{D}_{2} K \xi^{2}+\left[(K-1)(1-\theta) k+2 \delta \hat{D}_{1}\right] \xi \\
-\delta \hat{D}_{2} K(1-K) k=0
\end{array}
$$

We first consider a naïve expansion, in which all quantities other than $\delta$ are of order unity. Seeking an expansion of the form $\xi=\Omega_{0}+\Omega_{1} \delta+\mathcal{O}\left(\delta^{2}\right)$, where $\Re\left(\Omega_{0}\right)>0$, we obtain the leading-order equation

$$
2 \Omega_{0}^{3}+(K-1)(1-\theta) k \Omega_{0}=0,
$$

and thus

$$
\Omega_{0}^{2}=\frac{(1-\theta)(1-K)}{2} k
$$

Since $0<K<1$, this is consistent if and only if $\theta<1$, and the corresponding asymptotic expansion for $s=\xi^{2}-$ $k^{2}$ is

$$
s=\frac{(1-\theta)(1-K)}{2} k-k^{2}+\mathcal{O}(\delta)
$$

Figure 4 a illustrates how well (73) captures the behaviour of $s(k)$ as the Damköhler numbers become small. Although, with the choice of $D a_{1} / D a_{2}$ employed in this figure, the system is always stable for small wavenumbers, $s(k)$ is positive for intermediate wavenumbers $k \approx$ $(1-\theta)(1-K) / 4$ (which in this case gives $k \approx 0.125)$.

When $\theta \geq 1$, the naïve expansion is not consistent with the condition $\Re(\xi)>0$, so we need to seek alternative expansions in this regime. Motivated by the small- $k$ results (63) and (66), we define rescaled variables via $s=\delta^{2} \hat{s}$ and $k=\delta \hat{k}$, and thus $\xi=\delta \hat{\xi}$, and we seek an expansion of the form $\hat{\xi}=\hat{\xi}_{0}+\hat{\xi}_{1} \delta+\mathcal{O}\left(\delta^{2}\right)$. Substituting this expansion into (70), we obtain

$$
\hat{\xi}_{0}=\frac{\hat{D}_{2} K(1-K) \hat{k}}{(K-1)(1-\theta) \hat{k}+2 \hat{D}_{1}} .
$$

This expression for $\hat{\xi}_{0}$ remains finite for all $\hat{k}$ as long as $(K-1)(1-\theta) \geq 0$, and so since $0<K<1$ this expansion can be uniform in $\hat{k}$ only if $\theta \geq 1$; it thus complements the naïve expansion described above. The corresponding expression for $\hat{s}$ is

$$
\hat{s}=\left[\left(\frac{\hat{D}_{2} K(1-K)}{(K-1)(1-\theta) \hat{k}+2 \hat{D}_{1}}\right)^{2}-1\right] \hat{k}^{2}+\mathcal{O}(\delta) .
$$

When $\theta>1$, equation (75) successfully captures the behaviour of $s$, as illustrated in figure $4 \mathrm{c}$. In particular, for small $\hat{k}$ it predicts instability precisely when (65) holds, and as $\hat{k} \rightarrow \infty$ the growth rate decays as $\hat{s} \sim-\hat{k}^{2}$.

However, when $\theta=1$, equation (75) fails to capture the decay terms which determine the position of the maximum of $s$, and it is necessary to seek a different rescaling of $\xi$ and $k$. Under any such scaling, the term in (70) proportional to $\xi^{2}$ is asymptotically smaller than the term proportional to $\xi$; on the other hand, to obtain a nontrivial dependence of $\xi$ on $k$ it is necessary to include all three remaining terms. This motivates the scaling $\xi=\delta^{1 / 2} \bar{\xi}, s=\delta \bar{s}$ and $k=\delta^{1 / 2} \bar{k}$, and the expansion $\bar{\xi}=\bar{\xi}_{0}+\bar{\xi}_{1} \delta^{1 / 2}+\mathcal{O}(\delta)$ then leads to the depressed cubic equation

$$
\bar{\xi}_{0}^{3}+\hat{D}_{1} \bar{\xi}_{0}-q=0, \quad \text { where } \quad q=\frac{\hat{D}_{2} K(1-K)}{2} \bar{k}>0 .
$$

The real root may be found explicitly by Cardano's method, giving

$$
\begin{gathered}
\bar{s}=\left[\left(\sqrt{\frac{q^{2}}{4}+\frac{\hat{D}_{1}^{3}}{27}}+\frac{q}{2}\right)^{1 / 3}-\left(\sqrt{\frac{q^{2}}{4}+\frac{\hat{D}_{1}^{3}}{27}}-\frac{q}{2}\right)^{1 / 3}\right]^{2} \\
-\bar{k}^{2}+\mathcal{O}\left(\delta^{1 / 2}\right)
\end{gathered}
$$

When $\theta=1$, equation (77) successfully captures the behaviour of $s$, as illustrated in figure $4 \mathrm{~b}$.

In summary, we find that in the limit in which both Damköhler numbers become small while their ratio remains of order unity, the stability depends on the parameter $\theta$. For $\theta<1$, so that the surface tension depends principally on the surface concentration, instabilities occur at wavenumbers $k$ of order unity, corresponding to preferred wavelengths roughly an order of magnitude greater than the thickness $\eta$ of the surface layer, and can do so even when the system remains stable as $k \rightarrow 0$. In contrast, for $\theta \geq 1$ the preferred wavenumbers $k$ decrease along with the Damköhler numbers; thus the long-wave stability criterion (65) continues to capture the behaviour of the system, and the preferred wavelength of instabilities becomes much larger than the thickness of the surface layer.

\section{SUMMARY AND CONCLUSIONS}

We have described the construction of a new fluiddynamical model of coupled flow and solute transport 

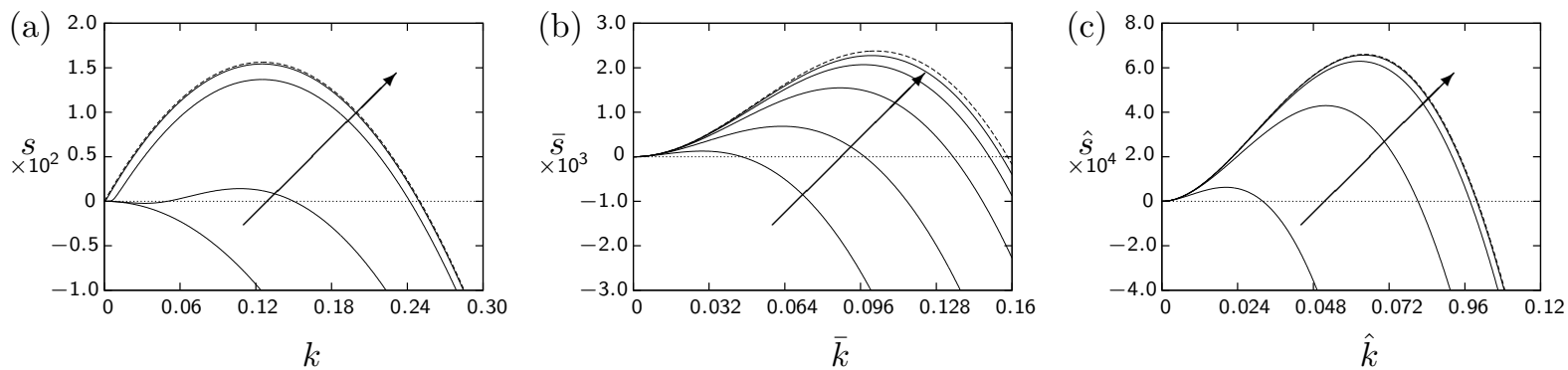

FIG. 4. (a) Perturbation growth rates $s(k)$ for $K=0.5$ and $\theta=0$, with $D a_{1} / D a_{2}=0.2$ and $D a_{2}=1,0.1,0.01$ and 0.001 (solid lines), together with the asymptotic result (73) (dashed lines). (b) Scaled perturbation growth rates $\bar{s}(\bar{k})$ for $K=0.5$ and $\theta=1$, with $D a_{1} / D a_{2}=0.1$ and $D a_{2}=1,0.1,0.01,0.001$ and 0.0001 (solid lines), together with the asymptotic result (77) (dashed lines). (c) Scaled perturbation growth rates $\hat{s}(\hat{k})$ for $K=0.5$ and $\theta=2$, with $D a_{1} / D a_{2}=0.1$ and $D a_{2}=1,0.1,0.01$ and 0.001 (solid lines), together with the asymptotic result (75) (dashed lines). The arrows show the direction of decreasing $D a_{2}$ in each case.

which naturally accommodates both classical surfactants and solutes with anti-surfactant properties. Under kinetic equilibrium between the free surface and the bulk, such models must agree with the surfacetension-concentration relationship described by the Gibbs isotherm (4) together with a suitable condition, such as the Henry isotherm, relating the equilibrium bulk and surface concentrations. When bulk-surface equilibrium does not hold, there is, in principle, freedom to extend the model in various ways. However, care must then be taken to distinguish artefacts of the extension from genuine physical phenomena, and in the model presented here we have included the parameter $\theta$, which allows this point to be investigated.

Considering the stability of an infinitely deep, initially quiescent layer of fluid suggests that, in contrast to surfactant solutions, anti-surfactant solutions may experience an instability driven by the accumulation of solute in the surface at points of surface flow convergence. The preferred spatial scales of this instability are rather small, but are sufficiently large relative to the thickness of the surface layer that the model remains consistent. For fast bulk-surface kinetics, for which the Damköhler numbers are of order unity, the parameter $\theta$ is irrelevant to the stability. For slower bulk-surface kinetics, $\theta$ plays a role in setting the spatial scale of the instability, and the version of the model for which surface tension depends solely on surface concentration (corresponding to $\theta=0$ ) predicts the shortest preferred wavelengths. This demonstrates that the precise formulation of the surface-tension law for anti-surfactants may have observable consequences, and deserves further investigation. It is possible, for example, that measurements using cantilever instruments could resolve the small-scale variations associated with the instability, while non-equilibrium surface-tension behaviour may also become apparent in the development of foams $[26]$.

The existence of a linear instability naturally raises the question of the state towards which the perturbed system evolves. Since this instability is essentially driven by per- turbations to the concentration fields, we may speculate that the first variable to evolve beyond the linear regime will be either the surface or the bulk concentration. The instability could be restrained by the breakdown of the linear bulk-surface flux or through changes to the transport rates; ultimately, it could manifest itself through precipitation of the solute in regions where the perturbed concentration exceeds the saturation concentration of the solute. One experimentally observable signature of this instability, therefore, might be a tendency for solutes to precipitate from solution in the vicinity of a free surface, under conditions when the bulk concentration is somewhat lower than its saturation value. An experimental investigation of this possibility would be of considerable interest.

Finally, we note that although the model presented here is consistent with the basic thermodynamics represented by the Gibbs isotherm, it remains essentially an extension of the established modelling framework for surfactants, and a gap still exists between fluid-dynamical models such as ours and more fundamental descriptions of salt solutions [12]. More sophisticated models, which take account of distinct species and their electrochemical interactions as well as appropriate non-equilibrium thermodynamics, may be required to bridge this gap.

\section{ACKNOWLEDGMENTS}

JJAC is supported by a University of Strathclyde Postgraduate Research Scholarship. SKW is supported by Leverhulme Trust Research Fellowship RF-2013-355.

\section{Appendix A: The infinite-depth limit of the finite-depth stability problem}

In this appendix we briefly discuss the finite-depth version of the stability problem with an undeformed free surface, focussing on the limit in which the depth of the 
fluid layer becomes infinite. We will demonstrate that the finite-depth problem is well posed for both surfactants and anti-surfactants, but that the only family of solutions available for surfactants becomes degenerate as the depth tends to infinity. A full investigation of finitedepth effects is ongoing.

For a layer of dimensionless depth $d^{*}$, the far-field conditions (28) are replaced by

$$
\mathbf{u}^{*}=\mathbf{0} \quad \text { and } \quad \frac{\partial c_{\mathrm{b}}^{*}}{\partial z^{*}}=0 \quad \text { on } \quad z^{*}=-d^{*} .
$$

In turn, the far-field conditions (46) are replaced by the conditions

$$
U\left(-d^{*}\right)=0, \quad W\left(-d^{*}\right)=0, \quad C^{\prime}\left(-d^{*}\right)=0,
$$

while the general solutions to (48) and (39) consistent with these boundary conditions become

$$
\begin{aligned}
& W\left(z^{*}\right)=\left(A_{1}+A_{2} \frac{z^{*}}{d^{*}}\right) \sinh \left(k\left(z^{*}+d^{*}\right)\right) \\
& -k d^{*}\left(A_{1}-A_{2}\right)\left(1+\frac{z^{*}}{d^{*}}\right) \cosh \left(k\left(z^{*}+d^{*}\right)\right)
\end{aligned}
$$

and

$$
C(z)=A_{3} \cosh \left(\xi\left(z^{*}+d^{*}\right)\right)
$$

where, as before, we have written $\xi=\sqrt{k^{2}+s}$. Note that we require that $\xi \neq 0$, i.e. $s \neq-k^{2}$, but impose no further condition on $\xi$. Henceforth we drop the star on $d^{*}$ for brevity.

The solvability condition becomes

$$
\left|\begin{array}{cccc}
k d \mathrm{c}(k d)-\mathrm{s}(k d) & -k d \mathrm{c}(k d) & 0 & 0 \\
2 k^{2} d \mathrm{c}(k d) & -\frac{2\left(1+k^{2} d^{2}\right)}{d} \mathrm{c}(k d)-2 k \mathrm{~s}(k d) & k \theta(1-K) \mathrm{c}(\xi d) & \frac{k(\theta-1)(K-1)}{K} \\
0 & 0 & \xi \mathrm{s}(\xi d)+D a_{2} K \mathrm{c}(\xi d) & -D a_{2} \\
K k^{2} d \mathrm{~s}(k d) & -\frac{K\left(1+k^{2} d^{2}\right)}{d} \mathrm{~s}(k d)-K k \mathrm{c}(k d) & -D a_{1} K \mathrm{c}(\xi d) & \xi^{2}+D a_{1}
\end{array}\right|=0
$$

where we have written s and $\mathrm{c}$ as shorthand for sinh and cosh respectively.

Solving (A5) numerically in the parameter regime (33), we typically find that if $0<K<1$ then instability is possible for a range of small values of $k$, as in the infinitedepth problem, whereas if $K>1$ then no instability occurs. A detailed discussion of the results for finite $d$ lies beyond the scope of this appendix; instead, here we will seek asymptotic results as $d \rightarrow \infty$. The form of the exponential terms in (A5) makes it natural to consider four distinguished limits, depending on the combination of $k, k d, \xi$ and $\xi d$ that is taken to remain finite and non-zero in this limit; we consider them in turn.

Case (i): $\Re(\xi)$ and $k$ remain finite and non-zero as $d \rightarrow \infty$. This is the case implicitly considered in $\S$ III by postulating an infinitely deep body of fluid. In this limit we may approximate all of the hyperbolic terms in (A5) by exponentials. We must consider the cases $\Re(\xi) \gtrless 0$ separately in order to discard the correct exponential terms; combining the results we find that the solvability condition (A5) reduces to

$$
\begin{aligned}
& 2 \xi^{3} \operatorname{sgn}(\xi)+2 D a_{2} K \xi^{2}+\left[(K-1)(1-\theta) k+2 D a_{1}\right] \operatorname{sgn}(\xi) \xi \\
& +D a_{2} K k(K-1)+\mathcal{O}\left(\mathrm{e}^{-2 k d}, \mathrm{e}^{-2 \operatorname{sgn}(\xi) \xi d}\right)=0, \quad(\mathrm{~A} 6)
\end{aligned}
$$

where $\operatorname{sgn}(\xi)= \pm 1$ if $\Re(\xi) \gtrless 0$.

If $\Re(\xi)>0$ then, as we have seen in $\S$ III C 1 , only the regime $0<K<1$ permits consistent solutions for long waves. Alternatively, if $\Re(\xi)<0$ then by defining $\xi^{\prime}=-\xi$ we again find that there are consistent solutions for long waves only when $0<K<1$. We conclude that when $K>1$, in order to find consistent solutions across all $k$ we must consider a different distinguished limit.

Case (ii): $\Re(\xi)$ and $k d$ remain finite and non-zero as $d \rightarrow \infty$. We now consider the possibility that $\xi$ remains of order unity (maintaining the possibility that $s=\mathcal{O}(1)$ ) as $d \rightarrow \infty$, but that this occurs only for very long waves. We thus define $\kappa=k d$ and set $\kappa=\mathcal{O}(1)$ as $d \rightarrow \infty$. Again considering $\Re(\xi) \gtrless 0$ separately, we reduce the solvability condition (A5) to

$$
\begin{aligned}
\left(\xi^{3}+\xi^{2} \operatorname{sgn}(\xi) D a_{2} K\right. & \left.+D a_{1} \xi\right)(\cosh (\kappa) \sinh (\kappa)-\kappa) \\
& +\mathcal{O}\left(\frac{1}{d}, \mathrm{e}^{-2 \operatorname{sgn}(\xi) \xi d}\right)=0
\end{aligned}
$$

Since $\xi \neq 0$ by assumption and the factor $\cosh (\kappa) \sinh (\kappa)-\kappa$ is strictly positive for $\kappa>0$, we conclude that $\xi$ must satisfy the quadratic equation $\xi^{2}+\xi \operatorname{sgn}(\xi) D a_{2} K+D a_{1}=0$. Again considering separately the cases $\operatorname{sgn}(\xi)= \pm 1$, we conclude that there are no consistent solutions in this distinguished limit for any positive value of $K$.

Case (iii): $\xi d$ and $k$ remain finite and non-zero as $d \rightarrow \infty$. We now define $\Xi=\xi d$, where $\Xi=\mathcal{O}(1)$. Approximating the hyperbolic terms in $k d$ by exponentials, 
we reduce the solvability condition (A5) to

$$
\begin{gathered}
{\left[k d^{2}(K-1)(1-\theta)+2 D a_{1} d^{2}+2 \Xi^{2}\right] \Xi \sinh (\Xi)} \\
+D a_{2} K d\left[(K-1) d^{2} k+2 \Xi^{2}\right] \cosh (\Xi)+\mathcal{O}\left(\mathrm{e}^{-2 k d}\right)=0 .
\end{gathered}
$$

As $d \rightarrow \infty$, the dominant terms are those in $d^{3}$, and so the solvability condition reduces to $\cosh (\Xi)=0$, with solutions $\Xi=\left(n+\frac{1}{2}\right) \pi$ i for $n \in \mathbb{Z}$. The solutions yield

$$
s \sim-k^{2}-\left(n+\frac{1}{2}\right)^{2} \frac{\pi^{2}}{d^{2}},
$$

which describe stable modes, independent of $K$ and decaying a little faster than the rate $s=-k^{2}$ set by the diffusion of a vertically constant perturbation. Crucially, when we take the limit of infinite depth, these modes collapse onto $s=-k^{2}$. The loss of these modes represents a degeneracy in the problem, which is important only if no other modes exist.

Case (iv): $\xi d$ and $k d$ remain finite and non-zero as $d \rightarrow \infty$. In this final case, we set $\Xi=\xi d$ and $\kappa=k d$ as before, and the solvability condition (A5) reduces to

$$
\begin{aligned}
& D a_{2} K(K-1) \kappa\left(\sinh ^{2}(\kappa)-\kappa^{2}\right) \cosh (\Xi) \\
+ & 2 D a_{1}(\cosh (\kappa) \sinh (\kappa)-\kappa) \Xi \sinh (\Xi)+\mathcal{O}\left(\frac{1}{d}\right)=0 .
\end{aligned}
$$

Rearranging then yields

$$
\Xi \tanh (\Xi) \sim \frac{D a_{2} K(1-K)}{2 D a_{1}} \frac{\kappa\left(\sinh ^{2}(\kappa)-\kappa^{2}\right)}{(\cosh (\kappa) \sinh (\kappa)-\kappa)}
$$

The function of $\kappa$ on the right-hand side is strictly positive for $\kappa>0$, so the sign of the right-hand side is identical to the sign of the factor $1-K$. Hence, it can be shown that for $0<K<1$ we obtain modes with $\Xi \in \mathbb{R}_{+}$and thus $s>-k^{2}$; these modes persist as $d \rightarrow \infty$, although they occur at wavelengths that scale with $d$, while the growth rates scale with $1 / d^{2}$. For $K>1$, we must seek imaginary solutions for $\Xi$. We may write $\Xi=\mathrm{i} \chi$ so that the left-hand side becomes $-\chi \tan (\chi)$, and so we obtain a spectrum of modes with $s \sim-\kappa^{2} / d^{2}-\chi^{2} / d^{2}<-k^{2}$.

The overall conclusion from this asymptotic analysis is that although the finite-depth stability problem is well posed for both surfactants and anti-surfactants, the limit $d \rightarrow \infty$ is degenerate. Only a particular family of modes survives in this limit, and this family is available only for anti-surfactants, $0<K<1$, for which it provides the dominant mode.

The modes that degenerate in the limit $d \rightarrow \infty$ do so because their spatial scale is naturally set by the depth of the layer, and becomes ill-defined in this limit. In contrast, the bulk concentration field for the nondegenerating modes has a boundary-layer structure and the depth of the layer becomes irrelevant. Since, from (39), the thickness of any concentration boundary layer must scale as $\xi=\sqrt{k^{2}+s}$, boundary layers can occur only when $\Re(s)>-k^{2}$, i.e. when the concentration perturbation is not decaying as rapidly as it would by diffusion alone. To resist this diffusive decay an instability mechanism must act near or at the surface, and thus perturbations with this structure are available only for anti-surfactants.
[1] D. J. Shaw, Introduction to Colloid and Surface Chemistry, 2nd ed. (Butterworths, 1970)

[2] A. P. dos Santos, A. Diehl, and Y. Levin, Langmuir 26, 10778 (2010)

[3] G. Vázquez, E. Alvarez, and J. M. Navaza, Journal of Chemical \& Engineering Data 40, 611 (1995)

[4] M. S. Borgas and J. B. Grotberg, Journal of Fluid Mechanics 193, 151 (1988)

[5] O. E. Jensen and J. B. Grotberg, Physics of Fluids A 5, 58 (1993)

[6] O. K. Matar and S. M. Troian, Physics of Fluids 11, 3232 (1999)

[7] G. Karapetsas, R. V. Craster, and O. K. Matar, Journal of Fluid Mechanics 670, 5 (2011)

[8] G. Karapetsas and V. Bontozoglou, Journal of Fluid Mechanics 741, 139 (2014)

[9] C.-H. Chang and E. I. Franses, Colloids and Surfaces A 100, 1 (1995)

[10] C. J. W. Breward, R. C. Darton, P. D. Howell, and J. R. Ockendon, Chemical Engineering Science 56, 2867 (2001)

[11] C. E. Morgan, C. J. W. Breward, I. M. Griffiths, P. D.
Howell, J. Penfold, R. K. Thomas, I. Tucker, J. T. Petkov, and J. R. P. Webster, Langmuir 28, 17339 (2012)

[12] P. B. Petersen and R. J. Saykally, Annual Review of Physical Chemistry 57, 333 (2006)

[13] T. Markovich, D. Andelman, and R. Podgornik, Europhysics Letters 106, 16002 (2014)

[14] More precisely, the Gibbs isotherm relates $\sigma^{\mathrm{eq}}$ to the chemical potential $\mu$ defined by $\mathrm{d} \mu=R T \mathrm{~d}(\log a)$, where $a$ is the thermodynamic activity, but for sufficiently dilute solutions $a$ is close to $c_{\mathrm{b}}$, and hence equation (4) holds.

[15] The conditions $1-K \lessgtr 0$ may be written as $K_{\mathrm{H}} \gtrless 0$, where $K_{\mathrm{H}}=-\eta(1-K)$. For surfactants, $K_{\mathrm{H}}>0$ is the equilibrium adsorption constant [9].

[16] A. Pereira and S. Kalliadasis, The European Physical Journal Applied Physics 44, 211 (2008)

[17] H. A. Stone, Physics of Fluids A 2, 111 (1990)

[18] H. Wong, D. Rumschitzki, and C. Maldarelli, Physics of Fluids 8, 3203 (1996)

[19] J. R. A. Pearson, Journal of Fluid Mechanics 4, 489 (1958) 
[20] I. Hashim and S. K. Wilson, Zeitschrift für angewandte Mathematik und Physik 50, 546 (1999)

[21] W. M. Haynes, ed., CRC Handbook of Chemistry and Physics, 94th ed. (CRC Press, Boca Raton, FL, 2013)

[22] S. Z. Mikhail and W. R. Kimel, Journal of Chemical \& Engineering Data 4, 533 (1961)

[23] K. R. Harris and P. J. Newitt, Journal of Physical Chemistry A 103, 6508 (1999)
[24] B. A. Noskov, Advances in Colloid and Interface Science 69, 63 (1996)

[25] S. E. Orchard, Journal of Applied Sciences Research 11, $451(1962)$

[26] I. Cantat, S. Cohen-Addad, F. Elias, F. Graner, R. Höhler, O. Pitois, F. Rouyer, A. Saint-Jalmes, R. Flatman, and S. Cox, Foams: Structure and Dynamics (Oxford University Press, 2013) doi: 10.1093/acprof:oso/9780199662890.001.0001 